\title{
Management of patients with urinary tract endometriosis by gynecologists
}

\author{
(D) Emsal Pınar Topdağı Yılmazı1, (D) Ömer Erkan Yapça1, (D) Gülşah Aynaoğlu Yıldız², (D) Yunus Emre Topdağı ${ }^{3}$, \\ (D) Fatih Özkaya ${ }^{4}$, (D) Yakup Kumtepe ${ }^{1}$ \\ 1Department of Obstetrics and Gynecology, Atatürk University Faculty of Medicine, Erzurum, Turkey \\ ${ }^{2}$ Clinic of Obstetrics and Gynecology, Nenehatun Maternity Hospital, Erzurum, Turkey \\ 3Department of Obstetrics and Gynecology, Sanko University Faculty of Medicine, Gaziantep, Turkey \\ 4Department of Urology, Atatürk University Faculty of Medicine, Erzurum, Turkey
}

\section{Abstract}

Objective: The aim was to report the postoperative outcomes of urinary tract endometriosis (UTE), which is a form of deep, infiltrative endometriosis, and to contribute to the literature by presenting our experience.

Material and Methods: In the present study, patients who underwent surgery for endometriosis at our clinic between 2005 and 2019 and had a final pathological diagnosis of UTE were examined in detail. Patient information was retrospectively retrieved from the medical records. Data obtained pre-, peri-, and postoperatively were analyzed.

Results: Mean age of the 70 patients included, according to the study criteria, was $32.73 \pm 7.09$ years. Ureteral involvement alone was observed in $49 \%(n=34)$ of the patients, bladder involvement alone was observed in $24 \%(n=17)$ of the patients, and both bladder and ureteral involvement were observed in $27 \%(n=19)$ of the patients. Microscopic hematuria was detected in $16 \%(n=11)$ of the patients, whereas preoperative urinary tract findings, such as recurrent urinary tract infections, were detected in $19 \%$ patients $(n=13)$. Of the patients, $56 \%$ ( $n=39)$ were identified with dyspareunia, 56\% $(n=39)$ with dysmenorrhea, and 30\% $(n=21)$ with pelvic pain. Visual analog scale score was significantly lower after the procedure $(\mathrm{p}<0.0001)$.

Conclusion: Although postoperative results were typically considered positive, surgical method performed in deep infiltrative endometriosis should aim to preserve fertility, improve quality of life, and reduce the complication rate to a minimum. (J Turk Ger Gynecol Assoc 2021; 22: 112-9)

Keywords: Urinary tract endometriosis, hematuria, dyspareunia, dysuria

Received: 13 April, 2020 Accepted: 13 October, 2020

\section{Introduction}

Endometriosis is a disease characterized by the localization of functional endometrial tissue outside the uterus, which often affects the genital organs $(1,2)$. Although it is most commonly reported among women in the reproductive age group, its exact prevalence is unknown. However, studies have shown that it is observed in the general population with a prevalence reported between $5 \%$ and $10 \%$ (3). The literature defines three types of endometriosis-superficial endometriosis, deep infiltrative endometriosis (DIE), and ovarian endometrioma (4). DIE is an aggressive form and is defined as an invasion of the peritoneum deeper than $5 \mathrm{~mm}$ (5). The intestines, urinary tract, uterosacral ligament, and vagina are often affected. The symptoms may become more apparent with increase in the depth of infiltration (6-8).

Although the reported prevalence of urinary tract endometriosis (UTE) is 1\%-6\%, this includes bladder and ureteral involvement, which is an aggressive form of DIE $(6,9)$. UTE is observed in 14\%-20\% of all patients with DIE (10). The bladder and ureter, which are the most frequently involved sites, are 
affected at ratios of $84 \%$ and $10 \%$, respectively (11-13). In UTE, which may be challenging to diagnose, endometriosis with bladder involvement should be considered if the patient describes frequent urination, cyclical bladder pain, dysuria, or hematuria (14). Endometriosis with ureteral involvement is typically asymptomatic and unaccompanied by genitourinary symptoms in $50 \%$ of patients. It is observed together with back and pelvic pain $(9,15,16)$. Magnetic resonance imaging (MRI) and ultrasonography (USG) exhibit a high specificity for endometriosis with bladder involvement in the diagnosis of UTE in which symptoms are highly non-specific. However, both techniques are inadequate in identifying lesions $<3 \mathrm{~cm}$ (17). Evidence concerning the diagnosis of endometriosis with ureteral involvement is extremely limited (2).

Laparotomy or laparoscopy can be preferred for surgical treatment of UTE in cases in which extremely limited medical approaches are available. However, laparotomy is not preferred due to the inability to completely view lesions, as well as lengthy hospitalization and greater blood loss. Nevertheless, despite the disadvantages of laparotomy, laparoscopy is typically not performed in the treatment of DIE (2).

The aim of this study was to present the treatment of patients with DIE characterized by bladder and ureteral involvement and its long-term outcomes. It was hoped that by presenting our experience in terms of effectiveness, safety, and complication rates of the most suitable approaches for the patient with UTE, characterized by bladder and ureter involvement, management of this challenging condition could be made somewhat simpler.

\section{Material and Methods}

This was a retrospective study, which was performed at the obstetrics and gynecology clinics of a university hospital between 2005 and 2019. All aspects of this research were approved by Ethics Committee of Atatürk University of Medical Sciences.

In this study patients who underwent surgery for endometriosis in our clinic between 2005 and 2019 and had a final pathological diagnosis of UTE were examined in detail. The pathological, clinical, and medical records were reviewed, and surgery type, complication rates, preoperative complaints, and duration of hospital stay were recorded. Patients with rectovaginal involvement, deep pelvic invasion, and complete or partial obliteration of the pouch of Douglas, together with an ovarian endometrioma were considered to have advanced stage (stage 3/4) disease according to the American Fertility Association scoring system (18). Based on their surgery records, patients with bladder and ureteral involvement who underwent ureterolysis, had partial or complete resection of the urethra, had bladder nodule resection, or had partial bladder resection were selected for detailed analysis.
From the medical records, it was determined whether the preoperative clinical evaluation of the patients was performed by a multidisciplinary team and whether the operating team was decided by establishing an expert committee, as well as the surgical approach used and the necessity of the imaging method used. All patients had undergone preoperative urinary USG, transvaginal USG, and renal function tests.

In cases with large ureteral nodules, the nodule was removed via the incision of the ureter and end-to-end anastomosis was performed. The bladder was sutured twice (Vicryl 3/0 or Monocryl 3/0) in cases wherein the bladder was completely opened. At the end of the procedure, $200-300 \mathrm{~mL}$ of $0.9 \% \mathrm{NaCl}$ solution diluted with indigo carmine was injected into the bladder for a fluid leakage test. Prophylactic antibiotics were administered to all patients preoperatively.

Postoperative follow-ups were performed at Atatürk University multidisciplinary endometriosis center-the tertiary referral center of the region-at the end of the first and sixth months. The management of patients who developed complications or who required recurrent surgery was performed by a multidisciplinary team. Preoperative and postoperative pain scores using a visual analog scale (VAS score) were obtained from medical records.

\section{Statistical analysis}

The analyses were performed using the SPSS, version 20 (IBM Inc., Armonk, NY, USA). Data are presented as mean, standard deviation, median, minimum, maximum, percentage, and number. Normal distribution of continuous variables was evaluated using the Shapiro-Wilk W test when the sample size was $<50$ and using the Kolmogorov-Smirnov test when it was $>50$. In the comparison of continuous variables with more than two independent groups, ANOVA test was used when distribution was normal, whereas the Kruskal-Wallis test was used in cases where it was not met. In $2 \times 2$ comparisons between categorical variables, the Pearson's chi-square test was used when the expected value was $>5$, Yates' chi-square test was used when the expected value was between 3 and 5 , and Fisher's exact test was used when the expected value was $<3$. In comparisons greater than $2 \times 2$ between categorical variables, the Pearson's chi-square test was used when the expected value was $>5$ and whereas the Fisher-FreemanHalton test was used when the expected value was $<5$. The level of statistical significance level was set as $p<0.05$.

\section{Results}

Seventy patients were identified who met the study inclusion criteria. The mean age of the patients was $32.73 \pm 7.09$ years. The surgical procedure was started laparoscopically in all patients. However, due to technical difficulties, laparotomy was initiated in 
three patients. Ureteral involvement alone was observed in $76 \%$ $(n=53)$ of the patients, bladder involvement alone was observed in $51 \%(\mathrm{n}=36)$ of the patients, and both bladder and ureteral involvement was observed in $27 \%(\mathrm{n}=19)$ of the patients.

Records showed that 29 (41\%) patients received medical treatment within the six months prior to surgery. Twenty nine of 70 patients had been prescribed $2 \mathrm{mg}$ of dienogest daily for 6 months as a medical treatment. Moreover, $16(23 \%)$ patients in the study group had undergone previous pelvic surgery (Table 1). During the preoperative period, 12 patients suspected of DIE received double J stents. However, two of these patients had ureteral injury despite this and underwent end-to-end anastomosis. Hydronephrosis was preoperatively diagnosed using preoperative imaging in eight patients with ureteral involvement. It was found that bilateral double $\mathrm{J}$ stent was placed by urologists prior to surgery in patients suspected of having severe ureteral involvement. In cases preoperatively suspected of having a bladder nodule, cystoscopy was performed to determine the localization of the nodule at the beginning of the process and to identify whether the ureteral orifices, and trigone in particular, were involved. Although it was not possible to obtain the intrinsic and extrinsic distinction of ureteral involvement from medical records, it was observed that this was evaluated in the final pathological assessment of

Table 1. Distribution of endometriotic nodules and patient symptoms

\begin{tabular}{|l|l|l|}
\hline \multirow{2}{*}{ Ureteral nodule } & No & $17(0.24)$ \\
\cline { 2 - 3 } & Yes & $53(0.76)$ \\
\hline \multirow{2}{*}{ Bladder nodule } & No & $34(0.49)$ \\
\cline { 2 - 3 } & Yes & $36(0.51)$ \\
\hline Both ureteral and bladder nodule & No & $\mathbf{1 9 ( 0 . 2 7 )}$ \\
\hline \multirow{2}{*}{ Microscopic hematuria } & Yes & $11(0.84)$ \\
\hline \multirow{2}{*}{ Recurrent urinary tract infection } & No & $57(0.81)$ \\
\cline { 2 - 3 } & Yes & $13(0.19)$ \\
\hline \multirow{2}{*}{ Dyspareunia } & No & $31(0.44)$ \\
\cline { 2 - 3 } & Yes & $39(0.56)$ \\
\hline \multirow{2}{*}{ Dysmenorrhea } & No & $31(0.44)$ \\
\cline { 2 - 3 } & Yes & $39(0.56)$ \\
\hline \multirow{2}{*}{ Dysuria } & No & $54(0.77)$ \\
\cline { 2 - 3 } & Yes & $16(0.23)$ \\
\hline \multirow{2}{*}{ Pelvic pain } & No & $49(0.7)$ \\
\cline { 2 - 3 } & Yes & $21(0.3)$ \\
\hline \multirow{2}{*}{ Preoperative medical treatment } & No & $41(0.59)$ \\
\cline { 2 - 3 } & Yes & $29(0.41)$ \\
\hline \multirow{2}{*}{ Previous pelvic surgery } & No & $54(0.77)$ \\
\cline { 2 - 3 } & Yes & $16(0.23)$ \\
\hline
\end{tabular}

the patient. It was determined that patients with severe ureteral involvement together with symptoms initially underwent ureterolysis, whereas those who developed severe fibrosis and irreversible stenosis underwent resection. In endometriosis with bladder involvement, it was determined that depending on the size of the nodule and infiltration, either the bladder was completely opened or partially resected or the nodule was excised. The medical records revealed that urologists were included in the surgery if deemed necessary by surgeons experienced in endometriosis. Moreover, the excision of bladder nodules and extrinsic ureteral nodules was mainly performed by gynecologists, although in cases with more severe complications, specialist urologists were included in the surgery. The bladder catheter remained in place for an average of 10 days, whereas the ureteral catheters remained for 4 to 6 weeks.

Microscopic hematuria was detected in $16 \%(n=11)$ of the patients, whereas preoperative urinary system findings, such as recurrent urinary tract infections, were detected in $19 \%(n=13)$ of the patients. Of the patients, $56 \%(n=39)$ were identified with dyspareunia, 56\% ( $\mathrm{n}=39)$ with dysmenorrhea, 16 with cyclical recurrent dysuria, and $30 \%(n=21)$ with pelvic pain. No significant difference was observed between the groups regarding CA-125 levels. Postoperative pain scores using a VAS was significantly lower after the procedure.

Examination of the operative notes of the patients revealed that ureterolysis was performed in 27 (39\%) patients, end-toend anastomosis was performed in $11(16 \%)$ patients, and no kidney loss was observed in any patient. During the followup period, four patients developed fistulas within 6 months; three of them had vesicovaginal fistula and one of them had rectovaginal fistula. Overall, five (7.1\%) patients in the study group were re-operated in our clinic within a maximum of 6 months postoperatively (Table 2). No significant difference was observed among the three study groups (ureteral, bladder, ureteral and bladder) in terms of their preoperative

Table 2. Distribution of urogynecological complications and surgical procedures employed

\begin{tabular}{|l|l|l|}
\hline \multicolumn{2}{|l|}{} & n (\%) \\
\hline \multirow{2}{*}{ Ureterolysis } & No & $43(0.61)$ \\
\cline { 2 - 3 } & Yes & $27(0.39)$ \\
\hline \multirow{2}{*}{ End-to-end anastomosis } & No & $59(0.84)$ \\
\cline { 2 - 3 } & Yes & $11(0.16)$ \\
\hline Ureterocystostomy & No & $70(0)$ \\
\hline \multirow{2}{*}{ Fistula (detected within 6 months) } & No & $66(0.94)$ \\
\cline { 2 - 3 } & Yes & $4(0.06)$ \\
\hline \multirow{2}{*}{ Re-operation within a maximum of 6 months } & No & $65(0.93)$ \\
\cline { 2 - 3 } & Yes & $5(0.07)$ \\
\hline
\end{tabular}


symptoms, urogram results, the medical treatment received for endometriosis preoperatively, and previous pelvic surgery (Table 3). When intra- and postoperative complications were examined, ureterolysis was significantly higher in the group with ureteral involvement. Ureterolysis was statistically significantly higher in both ureteral and bladder nodule group than bladder nodule group $(p=0.016$; Table 4$)$. The upper limit for the CA-125 level was considered $35 \mathrm{IU} / \mathrm{mL}$. When the groups were examined with respect to their CA-125 levels, the mean concentrations were $113 \pm 165 \mathrm{IU} / \mathrm{mL}$ in the group with ureteral involvement, $117 \pm 232 \mathrm{IU} / \mathrm{mL}$ in the group with bladder involvement, and $94 \pm 56 \mathrm{IU} / \mathrm{mL}$ in the group with both ureteral and bladder involvement which was not significantly different (Table 5). Furthermore, nine patients presented to the in vitro fertilization unit of our clinic within six months and that seven of them became pregnant.

The patients were questioned in detail especially when they were called for follow-up in terms of preoperative symptoms. Upon comparing the severity of symptoms of the patients at the end of the sixth postoperative month, the severity was reduced at least by half in $75 \%$ of the patients. When the postoperative pain scores using a VAS score were compared

Table 3. Comparison of symptoms according to endometriotic nodule localization

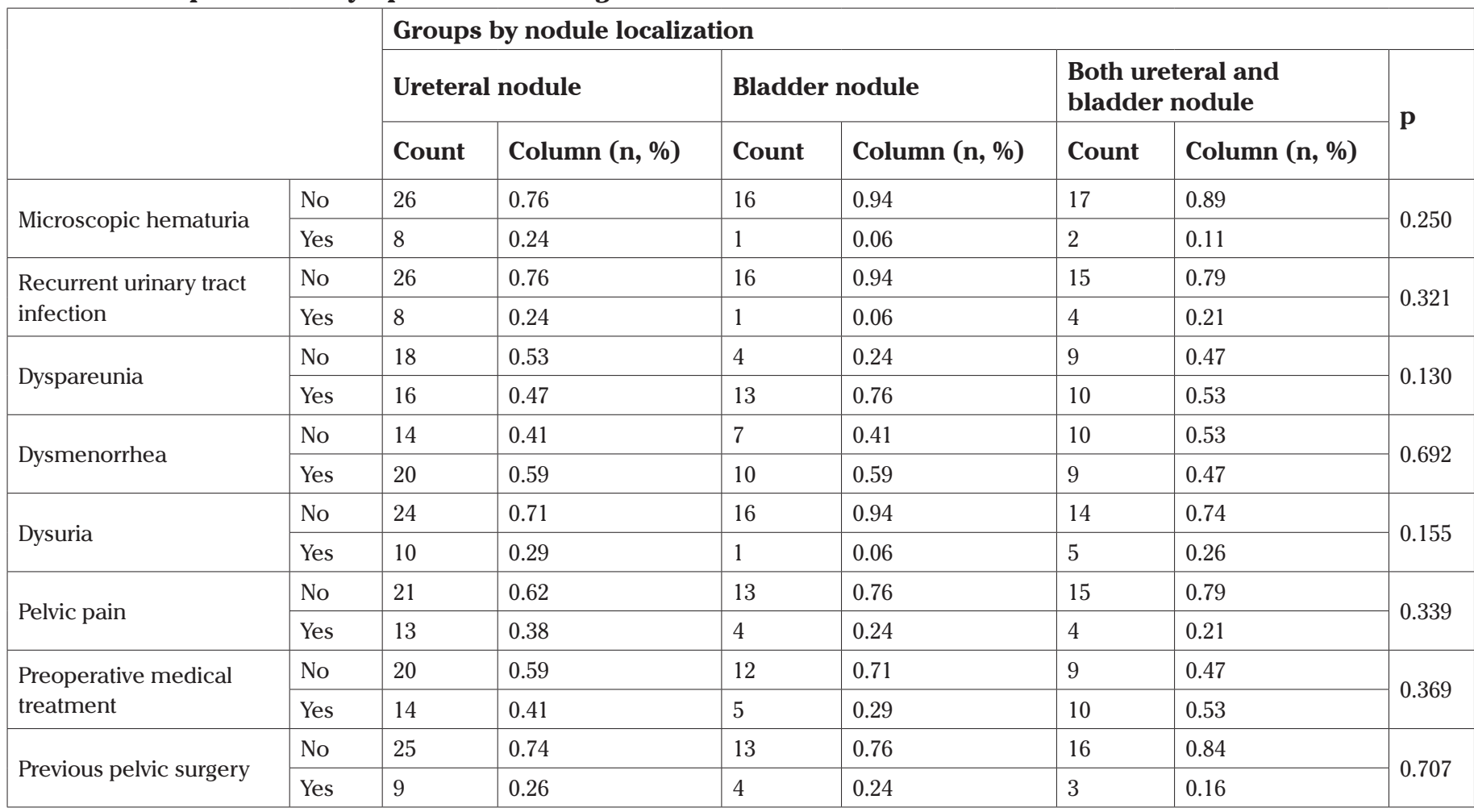

Table 4. Comparison between groups in terms of the complications of treatment approaches

\begin{tabular}{|c|c|c|c|c|c|c|c|c|}
\hline & & \multicolumn{7}{|c|}{ Groups according to nodule localization } \\
\hline & & \multicolumn{2}{|c|}{ Ureteral nodule } & \multicolumn{2}{|c|}{ Bladder nodule } & \multicolumn{2}{|c|}{$\begin{array}{l}\text { Both ureteral and bladder } \\
\text { nodule }\end{array}$} & \multirow{2}{*}{$\mathbf{p}$} \\
\hline & & Count & Column (n, \%) & Count & Column (n, \%) & Count & Column (n, \%) & \\
\hline \multirow{2}{*}{ Ureterolysis } & No & 20 & 0.59 & 15 & 0.88 & 8 & 0.42 & \multirow{2}{*}{$0.016^{*}$} \\
\hline & Yes & 14 & 0.41 & 2 & 0.12 & 11 & 0.58 & \\
\hline \multirow{2}{*}{ End-to-end anastomosis } & No & 29 & 0.85 & 15 & 0.88 & 15 & 0.79 & \multirow{2}{*}{0.761} \\
\hline & Yes & 5 & 0.15 & 2 & 0.12 & 4 & 0.21 & \\
\hline \multirow{2}{*}{ Urogynecological fistulas } & No & 33 & 0.97 & 16 & 0.94 & 17 & 0.89 & \multirow{2}{*}{0.567} \\
\hline & Yes & 1 & 0.03 & 1 & 0.06 & 2 & 0.11 & \\
\hline \multirow{2}{*}{$\begin{array}{l}\text { Re-operation within a } \\
\text { maximum of } 6 \text { months }\end{array}$} & No & 30 & 0.88 & 16 & 0.94 & 19 & 1.00 & \multirow{2}{*}{0.296} \\
\hline & Yes & 4 & 0.12 & 1 & 0.06 & 0 & 0.00 & \\
\hline
\end{tabular}


with preoperative scores, the mean pain score was 5 before the procedure and this had reduced to 2 in the sixth month after surgical or medical treatment. VAS score was significantly lower after the procedure $(\mathrm{p}<0.0001)$ (Table 6). According to our medical records, patients did not die in the long term and did not apply to our urology or obstetrics and gynecology clinic with the same symptoms.

\section{Discussion}

Several studies in the literature have shown that DIE surgery, which has a high probability of complications, requires a multidisciplinary approach both in the pre- and postoperative periods and that it should be performed by an experienced team specialized in the field $(19,20)$. Our study demonstrated that a multidisciplinary approach ensures an improvement in the quality of life and a significant reduction in pain. Postoperative pain scores using a VAS score was significantly lower after the procedure. With regard to this disease, for which rather different views have been reported in terms of treatment approaches, it is crucial that the surgical approaches employed for preventing recurrence and ensuring patient comfort primarily include the complete excision of nodules (19). In our study, $41 \%$ of patients had previously received medical treatment that was ineffective and that complete excision of the nodules was effective. Our higher rate of UTE, which is higher compared with that reported in the literature, can be attributable to the fact that our center is the largest endometriosis clinic in the region and that most of the patients were diffuse, advanced, and serious cases at the time of diagnosis $(21,22)$. The operative notes of the patients who developed fistula showed that harmonic scalpel was used during dissection. Because the heat effect generated in the surrounding tissues by the energy can lead to thermal damage in the lateral tissues with the increase in operation time and temperature, it was considered as a factor in the occurrence of the damage.
Endometriosis with bladder involvement, which is observed in approximately $12 \%$ of patients with DIE, is characterized by a partial or complete involvement of the detrusor muscle layers $(20,21)$. This may be owing to the fact that the detrusor muscle is stronger than ureteral muscles and has a denser vascular network that contributes to the healing process (22). The incidence of bladder involvement, which is a rare form of DIE, reported in the literature has gradually increased (19). It has been suggested that this may have an iatrogenic origin and the increased prevalence of endometriosis with bladder involvement might be particularly due to the increased rate of cesarean surgeries. It has been shown that $50 \%$ of endometriosis with bladder involvement occurs in women who have undergone a previous surgery (23). In our case series, $23 \%$ of the patients with UTE had undergone previous pelvic surgery. Four of our patients with bladder involvement had previously undergone pelvic surgery.

Studies have shown that the distal ureter is more frequently involved due to its proximity to the posterior compartment of the pelvic organs (24). Therefore, endometriosis, the pathophysiology of which remains unclear, has been associated with retrograde menstruation (2), and it is thought to be caused by the accumulation of menstrual blood and endometrial cells in the pelvis due to gravity and their presence at that location for a prolonged time (25). Based on this theory, it has been shown that rectovaginal involvement is more common than bladder involvement (26). Although 90\% of women have retrograde menstruation, the reason for $10 \%$ of them developing endometriosis remains unclear. However, the effect of gravity, more frequent occurrence of endometriosis on the left side, and rarer occurrence of endometriosis with bladder involvement in the retroverted uterus, as well as the fact that menstrual blood remains more on the left side due to the sigmoid colon may provide an explanation for this theory remaining the most likely $(2,20)$. In the study of Schonman et al. (19) half of the patients with endometriosis with bladder involvement had an ovarian

Table 5. Relationship between location of endometriotic nodules in the urogenital region and CA-125

\begin{tabular}{|c|c|c|c|c|c|}
\hline & \multicolumn{4}{|l|}{ CA-125 } \\
\hline & & Mean \pm SD & Median (min-max) & Chi-square & $\mathbf{p}$ \\
\hline \multirow{2}{*}{$\begin{array}{l}\text { Groups according to nodule } \\
\text { location }\end{array}$} & Ureteral nodule & $113 \pm 165$ & $78(7-961)$ & \multirow{2}{*}{3.635} & \multirow{2}{*}{0.162} \\
\hline & Both ureteral and bladder nodule & $94 \pm 56$ & $78(19-197)$ & & \\
\hline
\end{tabular}

Table 6. Comparison of VAS scores before and six months after treatment

\begin{tabular}{|l|l|l|l|}
\hline Variable & $\begin{array}{l}\text { Before treatment }(\mathbf{n}=\mathbf{7 0}) \\
\text { Median (min-max) }\end{array}$ & $\begin{array}{l}\text { Six months after treatment }(\mathbf{n}=\mathbf{7 0}) \\
\text { Median (min-max) }\end{array}$ & $\mathbf{p}$ \\
\hline Postoperative pain score (VAS) & $5(4-10)$ & $2(3-5)$ & 0.033 \\
\hline VAS: Visual analog scale, min: minimum, max: maximum & & \\
\hline
\end{tabular}


endometrioma and almost one-third of them had rectosigmoid lesions. Kovoor et al. (21) reported that $76 \%$ of patients with DIE were associated with deep endometriotic lesions in the pelvis, including rectovaginal, parametrial, and uterosacral nodules. Moreover, we found that ureteral involvement was higher than bladder involvement in UTE.

Endometriosis with ureteral involvement is a component of DIE. It is often non-symptomatic and complaints of the patients are non-specific. Patients typically describe complaints related to lateral pain and cystitis (22). Reportedly, 30\% of patients remained asymptomatic and the diagnosis was incidental (27). At the time of diagnosis, decreased renal function can be observed in approximately one-third (30\%) of patients and kidney loss may be present in 25\%-43\% patients (28). In the study conducted by Cavaco-Gomes et al. (29) on 700 patients with endometriosis with ureteral involvement, they reported no urinary system symptoms. They also found that $81 \%$ of patients described dysmenorrhea, $70 \%$ described pelvic pain, and $66 \%$ described dyspareunia (29). Schonman et al. (19) showed in their case series that $40 \%$ of their patients had urinary complaints. In our case series, 39 (56\%) patients presented with complaints of dyspareunia, 39 (56\%) patients with complaints of dysmenorrhea, and 21 (30\%) with complaints of pelvic pain. In addition, one (16\%) patient had microscopic hematuria and 13 (19\%) patients had urinary tract symptoms, such as recurrent urinary tract infection. No renal loss was detected in any patient.

In the treatment of endometriosis, whether an aggressive surgical procedure or conservative approach will be preferred may vary depending on the preoperative evaluation of the patient, the experience of the surgeon, and the severity of the symptoms. Preoperative diagnosis is extremely controversial due to variations in the scope of lesions. USG and MRI have high specificity in detecting endometriosis with bladder involvement, but both are insufficient in identifying lesions $<3 \mathrm{~cm}$ (17). Nezhat et al. (2) reported that they initially plan cystoscopy simultaneously with USG and laparoscopy prior to surgery when they suspect endometriosis with bladder involvement. Various studies have demonstrated that pelvic MRI achieves a sensitivity of up to $88 \%$ and specificity of up to $99 \%$ in the diagnosis of endometriosis with bladder involvement and that it has a diagnostic accuracy of approximately $98 \%$ (27). In our study, preoperative imaging methods were used depending on the severity of the disease and that USG and concurrent cystoscopy were performed in patients suspected of severe UTE. MRI was performed preoperatively in $80 \%$ of patients with UTE.

The primary clinical goals are to recover the damaged ureter, prevent kidney loss, and reduce patient complaints. Seracchioli et al. (30) successfully performed laparoscopic ureterolysis in 22 of 30 patients with endometriosis with ureteral involvement and treated eight other patients by performing ureteroureterostomy or ureteroneocystostomy. However, despite all these interventions, unpredictable complications, such as fistula, leakage after end-to-end anastomosis, and leakage after reimplantation, can be postoperatively observed (22). In their study, Rozsnyai et al. (31) reported complications in four patients treated for endometriosis with ureteral involvement and in two patients treated for endometriosis with bladder involvement. These complications were ureteric fistula due to thermal damage caused by harmonic scalpel and pyelonephritis, bladder atony, and vesicovaginal fistula following ureteroneocystostomy (31). During the postoperative period, recurrent surgery was necessary in five patients, vesicovaginal fistula in three patients, and rectovaginal fistula in one patient within 6 months.

Currently, the treatment of endometriosis with bladder involvement remains a controversial subject. Treatment may depend on various factors including the patient age, fertility status, disease prevalence, and symptom severity as well as presence of other pelvic lesions. Medical treatment may be preferred, depending on disease severity, but the high recurrence rates and symptom recurrence upon treatment discontinuation must be considered (19). However, as endometriosis is a progressive disease, following the surgical excision of endometriotic nodules, hormonal treatment can be administered to prevent recurrence and reduce pain (32).

In their study, Fedele et al. (33) demonstrated that even if medically induced amenorrhea is achieved, endometriotic nodules regress by only $30 \%$. Smooth muscle fibers and fibrosis constitute $60 \%$ of the histopathological component of the nodule that does not respond to hormonal treatment, whereas the remaining tissue is the endometrial epithelium (34). Price et al. (23) argued for both medical and surgical approaches in treatment, but observed surgical excision to be more effective. In our study, a significant portion of the patients had previously received hormonal treatment, and the surgical treatment approach had become inevitable thereafter. We believe that a laparoscopic approach performed by experienced surgeons is the preferred treatment method for both ureteral and bladder involvement in severe cases of endometriosis, and our study shows that long-term outcomes are extremely satisfactory.

\section{Conclusion}

The surgical method used in DIE, the pathophysiology of which is not understood and the optimal treatment methods remain unclear, should aim to preserve fertility, improve the quality of life, and reduce the complication rate to a minimum. We also recommend performing abdominal ultrasound in all DIE patients prior to surgery. This study 
has shown that the long-term outcomes of this challenging surgical procedure performed by experienced surgeons are extremely satisfactory.

Ethics Committee Approval: All aspects of this research were approved by Ethics Committee of Atatürk University of Medical Sciences.

Informed Consent: All participants had completed informed consent before surgery.

Peer-review: Externally peer-reviewed.

Author Contributions: Surgical and Medical Practices: E.P.T.Y., Ö.E.Y.; Concept: G.A.Y., Y.E.T.; Design: E.P.T.Y., Y.E.T.; Data Collection or Processing: Ö.E.Y., Y.E.T., F.Ö.; Analysis or Interpretation: E.P.T.Y., Ö.E.Y., F.Ö., Y.K.; Literature Search: E.P.T.Y., G.A.Y.; Writing: E.P.T.Y., Y.E.T.

Conflict of Interest: No conflict of interest is declared by the authors.

Financial Disclosure: The authors declared that this study received no financial support.

\section{References}

1. Freire MJ, Dinis PJ, Medeiros R, Sousa L, Águas F, Figueiredo A Deep Infiltrating Endometriosis-Urinary Tract Involvement and Predictive Factors for Major Surgery. Urology 2017; 108: 65-70.

2. Nezhat C, Falik R, McKinneyS, King LP. Pathophysiology and management of urinary tract endometriosis. Nat Rev Urol 2017; 14: $359-72$.

3. Eskenazi B, Warner ML. Epidemiology of endometriosis. Obstet Gynecol Clin North Am 24 1997; 24: 235-58.

4. Tosti C, Pinzauti S, Santulli P, Chapron C, Petraglia F. Pathogenetic Mechanisms of Deep Infiltrating Endometriosis. Reprod Sci 2015; 22: 1053-9.

5. Koninckx PR, Meuleman C, Demeyere S, Lesaffre E, Cornillie FJ. Suggestive evidence that pelvic endometriosis is a progressive disease, whereas deeply infiltrating endometriosis is associated with pelvic pain. Fertil Steril 1991; 55: 759-65.

6. Veeraswamy A, Lewis M, Mann A, Kotikela S, Hajhosseini B, Nezhat C. Extragenital endometriosis. Clin Obstet Gynecol 2010; 53: 449-66.

7. Nezhat C, Nezhat F, Nezhat C (edts). Nezhat's Video-assisted and robotic-assisted laparoscopy and hysteroscopy. UK: Cambridge Uni Press; 2013. p. 265-302.

8. Nezhat C, Winer WK, Nezhat F, Nezhat C. Laparoscopic treatment of endometriosis with laser and videocamera augmentation (videolaseroscopy). J Gynecol Surg 2009; 5: 163-9.

9. Gustilo-Ashby AM, Paraiso MF. Treatment of urinary tract endometriosis. J Minim Invasive Gynecol 2006; 13: 559-65.

10. Knabben L, Imboden S, Fellmann B, Nirgianakis K, Kuhn A, Mueller MD. Urinary tract endometriosis in patients with deep infiltrating endometriosis: prevalence, symptoms, management, and proposal for a new clinical classification. Fertil Steril 2015; 103: 147-52.

11. Berlanda N, Vercellini P, Carmignani L, Aimi G. Amicarelli F, Fedele L. Ureteral and vesical endometriosis. Two different clinical entities sharing the same pathogenesis. Obstet Gynecol Surv 2009; 64: 83042.

12. Abeshouse BS, Abeshouse G. Endometriosis of the urinary tract: a review of the literature and a report of four cases of vesical endometriosis. J Int Coll Surg 1960; 34: 43-63.

13. Antonelli A, Simeone C, Zani D, Sacconi T, Minini G, Canossi $\mathrm{E}$, et al. Clinical aspects and surgical treatment of urinary tract endometriosis: our experience with 31 cases. Eur Urol 2006; 49: 1093-7.

14. Antonelli A. Urinary tract endometriosis. Urologia 2012; 79: 167-70.

15. Comiter CV. Endometriosis of the urinary tract. Urol Clin North Am 2002; 29, 625-35.

16. Cavaco-Gomes J, Martinho M, Gilabert-Aguilar J, Gilabert-Estélles J. Laparoscopic management of ureteral endometriosis: a systematic review. Eur J Obstet Gynecol Reprod Biol 2017; 210: 94-101.

17. Kołodziej A, Krajewski W, Dołowy Ł, Hirnle L. Urinary tract endometriosis. Urol J 2015; 12: 2213-7.

18. Adamson GD, Pasta DJ. Endometriosis fertility index: the new, validated endometriosis staging system. Fertil Steril 2010; 94: 160915.

19. Schonman R, Dotan Z, Weintraub AY, Bibi G, Eisenberg VH, Seidman DS, et al. Deep endometriosis inflicting the bladder: longterm outcomes of surgical management. Arch Gynecol Obstet 2013; 288: 1323-8.

20. Berlanda N, Vercellini P, Carmignani L, Aimi G, Amicarelli F, Fedele L. Ureteral and vesical endometriosis. Two different clinical entities sharing the same pathogenesis. Obstet Gynecol Surv 2009; 64: 83042.

21. Kovoor E, Nassif J, Miranda-Mendoza I, Wattiez A. Endometriosis of bladder: outcomes after laparoscopic surgery. J Minim Invasive Gynecol 2010; 17: 600-4.

22. Darwish B, Stochino-Loi E, Pasquier G, Dugardin F, Defortescu G, Abo C, et al. Surgical Outcomes of Urinary Tract Deep Infiltrating Endometriosis. J Minim Invasive Gynecol 2017; 24: 998-1006.

23. Price DT, Maloney KE, Ibrahim GK, Cundiff GW, Leder RA, Anderson EE. Vesical endometriosis: report of two cases and review of the literatüre. Urology 1996; 48: 639-43.

24. Yohannes P. Ureteral endometriosis. J Urol 2003; 170: 20-5.

25. Dmowski WP, Radwanska E. Current concepts on pathology, histogenesis and etiology of endometriosis. Acta Obstet Gynecol Scand Suppl 1984; 123: 29-33.

26. Abrão MS, Podgaec S, Dias JA, Averbach M, Silva LF, Marino de Carvalho F. Endometriosis lesions that compromise the rectum deeper than the inner muscularis layer have more than $40 \%$ of the circumference of the rectum affected by the disease. J Minim Invasive Gynecol 2008; 15: 280-5.

27. Maccagnano C, Pellucchi F, Rocchini L, Ghezzi M, Scattoni V, Montorsi F, et al. Diagnosis and treatment of bladder endometriosis: state of the art. Urol Int 2012; 89: 249-58.

28. Stillwell TJ, Kramer SA, Lee RA. Endometriosis of the ureter. Urology 1986; 28: 81-5.

29. Cavaco-Gomes J, Martinho M, Gilabert-Aguilar J, Gilabert-Estélles J. Laparoscopic management of ureteral endometriosis: A systematic review. Eur J Obstet Gynecol Reprod Biol 2017; 210: 94-101.

30. Seracchioli R, Mabrouk M, Manuzzi L, Guerrini M, Villa G, Montanari $\mathrm{G}$, et al: Importance of retroperitoneal ureteric evaluation in cases of deep infiltrating endometriosis. J Minim Invasive Gynecol 2008; 15: 435-9.

31. Rozsnyai F, Roman H, Resch B, Dugardin F, Berrocal J, Descargues $\mathrm{G}$, et al; CIRENDO Study Group. Outcomes of surgical management of deep infiltrating endometriosis of the ureter and urinary bladder. JSLS 2011; 15: 439-47. 
32. Donnez J, Squifflet J. Complications, pregnancy and recurrence in a prospective series of 500 patients operated on by the shaving technique for deep rectovaginal endometriotic nodules. Hum Reprod 2010; 25: 1949-58.

33. Fedele L, Bianchi S, Zanconato G, Tozzi L, Raffaelli R. Gonadotropinreleasing hormone agonist treatment for endometriosis of the rectovaginal septum. Am J Obstet Gynecol 2000; 183: 1462-7.
34. Bazot M, Malzy P, Cortez A, Roseau G, Amouyal P, Daraï E. Accuracy of transvaginal sonography and rectal endoscopic sonography in the diagnosis of deep infiltrating endometriosis. Ultrasound Obstet Gynecol 2007; 30: 994-1001. 